\title{
The summary of Complex scene illumination direction estimating method
}

\author{
Xue Leng ${ }^{1,}$, Jinhua Yang $^{1}$ and Yu Miao ${ }^{2, *}$ \\ ${ }^{1}$ School of Photoelectric Engineering, Changchun University of Science and Technology, \\ Changchun, 130000, China \\ ${ }^{2}$ School of Computer Science, Changchun University of Science and Technology, Changchun, \\ 130000, China \\ aLengxue79@126.com.
}

Keywords: Augmented reality Illumination consistency Illumination direction.

\begin{abstract}
Augmented reality technology is to expand the virtual reality technology. It can make virtual objects and real scene closely integrated. It can enhance the user's understanding of the real environment. Illumination consistency is an important aspect of the fuse of virtual objects and real scene. In order to achieve consistency of complex scene illumination. In this paper, we start from the complex scene lighting features. Study and compare principles, characteristics and scope of use of all kinds of illumination direction estimation method.
\end{abstract}

\section{Introduction}

Augmented reality technology is an important branch of the virtual reality technology. It use computer graphics and visualization technologies to produce virtual objects. By using the sensor technology will place virtual objects in the real world. With the display device will integrate the virtual and real environment objects. Enhanced the real scene. Illumination estimation of complex scenes is an important research topic in the field of computer vision. It is in the target tracking, augmented reality and other fields have important applications. In augmented reality technology Vitual objects share the same scene with real lighting conditions. The appearance of the vitual object changes should be consistent with the objects in the real scene. When the illumination of the real scene changes, Illumination of the vitual objects also have corresponding changes. In order to achieve the illumination consistency of complex scene, require real-time estimation the illumination direction.

\section{The light characteristics of complex scenes}

The main source of complex scenes are skylight and sunlight. When the sunlight through the atmosphere, it can be scattered, refracted, bsorbed and formed shylight. Sunlight and skylight reflected through the ground and objects. It can formed ambient light. Sun light is the important nature light. It plays a decisive role of the complex scenes illumination. Since the distance between the sun and earth is very far. So for a point on the ground, the sun is a surface light source with a small solid angle. Therefore sunlight can be modeled as a parallel light source. Because sunlight transmission will lose some energy in the atmosphere, Therefore when analyzing the issue, only consider the incident to light after the intensity of sunlight through the atmosphere of the scene. When sunlight passes through the atmosphere, it is scattered or refracted by the molecules and dust in the atmosphere. These formed skylight. So the skylight is a surface light. Different particles have different scattering effect. By analysis of sunlight and skylight characteristic, it can be seen lighting conditions in complex scene is very complex. Skylight and sunlight will be great changes, when they are at different times, especially when the clouds drifting across. Luminance and chrominance variations are belong to the dynamic changes.Figure1 is a schematic view of a complex environmental model. 




Figure1 schematic view of a complex environmental model

\section{Illumination direction estimation}

Most researchers study the direction of illumination is limited to the simple scene. That is, they research a single light. Geometry, textures, materials and other information objects in the scene are known. Study on the illumination direction estimation of complex scenes are less. In this paper, we will classify and summary the methods of illumination estimation in the complex scene.

Method classification. According to the researchers whether by means of calibration object, the illumination direction estimation method can be divided into two categories. One method is using a calibration, the other method does not require calibration object. Among them, the estimation method does not require calibration object can be subdivided into three-dimensional visual methods and artificial intelligence.

The method of light measure ball. Early investigators were obtainen illumination parameters by light measure ball. Light measure ball radius are known. Zhang yufei use the least squares method and the iterative method to determine the critical point on the light measure ball dimensional geometry information. Then estimate the illumination direction and light intensity of the light source in the scene and other parameters. Bouganis C.S using light measure ball having Lambert surface reflective properties as the calibration object. The light measure ball image is divided into many sub-region. Each sub-region corresponds to a virtual light source. Use the difference of adjacent sub-region virtual light sources to estimate parameters. This method has good robustness and high precision. Feng yan use two light measure ball having Labertian surface reflection characteristics as a calibration object. By image analysis and spatial analytic geometry. Estimate the illumination parameters of the scene.

Zhou wei use light measure ball having a diffuse and specular reflection characteristic, by stereo image pairs obtained radius and central location of light measure ball. Then separated the diffuse component and the specular component of the light measure ball. Used a bright surface light measure ball, through the method of specular reflection surface matching error minimization, it is able to estimate the size and location information of a scene in a plurality of surface light source. This method can simultaneously determine the direction of light and light intensity of a plurality of light sources. Zhou wei build a general framework to estimate the scene lighting parameters. It can simultaneously estimate the scene illumination parameters of a plurality of different types of light sources. Using the light measure ball method needs to know the ball's geometry and surface reflection properties. The light measure ball is not portable, and the light measure ball will appear in the scene image. If the view mobile, scenario will change too. Then it need to collect the image of the light measure ball again to estimate the illumination parameters of scene. So this kind of method has some limitations in use.

Stereo vision method. Stereo vision method can avoid the disadvantages of light measure ball method, using the scene of light and shade and shadow or local geometric information, estimates illumination parameters of the scene.

Zheng qinfeng assumes that objects in the scene for the lambert. Using the light and shade information of image contour, estimate the deflection and obliquity of the light source. Rebuild the three-dimensional shape of the object. M. Gingham detectes the illumination in the scene using the shadows in the scene and the corresponding relationship between the feature points. Determines the 
three-dimensional space coordinates. But this method is only suitable for single light in simple scenario. And the object's shadow projected onto a flat surface.

Sato I uses the shadow cast by incident light, estimate the illumination distribution of complex scenes. But the approach requires the shape of the two objects are known. And accept the shadow casting object surface reflectivity is known. Wang yang according to the known scene geometry information, combined with the scene of light and shade and shadow information can estimate the multiple direction of light source light direction from a single scene image. Li yuanzhen using scene of light and shade, shadow and highlight areas, he put forward a new kind of estimation algorithm, it does not need to assume that the object surface feature. This method can be used for illumination recovery in the texture of the scene. Yao yuan proposed a method of scene light real-time detection using a normal position mark. Although this method can locate the light source, estimate the light intensity and color of the light source. But the method depends on the location in the scene. So it is only applicable to simple scenario of vitual light source.

For complex scenarios of augmented reality system, the scene will change as the user viewpoint move. The information in the scene is difficult to obtain accurately.

The method of artificial intelligence. Tian yingli puts forward the method of using neural network to estimate the illumination parameters. This method needs for training neural networks. When the viewpoint moves, it needs to collect images calibration objects for training.so the computational complexity greatly. But this method is suitable for the existence of multiple light sources in the scene. The mixed reflection model is put forward by S.Y.Cho.it used to estimate the scene illumination parameters. It use of the two kinds of neural network, these two kinds of neural network also need training. So it is hard to meet the requirements of real-time. But this model is suitable for the objects in the scene surface reflectance is unknown, image contains more noise and there are strong specular reflection. S. Karungaru put forward the estimation method of combining genetic algorithm and neural network. But the method also applies only to a fixed point. When the point of view changes, it needs to be trained neural network. It can be applicable to the new scenario of network weights. C. K. Chow puts forward a regression network. It uses the surface input and the real value output.Combing scene depth information, it is able to estimate the scene illumination direction of high precision. However, the method used in the scene depth information is obtained by $3 \mathrm{~d}$ scanner. This increases the cost and complexity of the system. When the point of view changes, Scenario will change too. It also need to training neural networks. To get network weights is suitable for the new scene. P. Nillius puts forward a new method, according to the single imange estimate light direction. This method using color and edge information, extraction with lambert surface reflection object contour features. Estimate the corresponding to every single contour lighting direction, then use the Bayesian networks determine the direction of light with the highest probability. It is the light direction of the light source in the scene. This approach requires the extracted object contour must be closed. So using scope is limited. M Chantler proposed to simultaneously surface texture characteristics classification and light direction estimation of classifier. But it must be trained before use. It cannot estimate light direction in real-time when the scene dynamic change.

\section{Conclusion}

Most researchers estimate the illumination parameters of scene using the light measure ball with its geometry size and the surface reflectivity known. In order to improve the practicability of the algorithm of light direction estimation. The reserchers study the new illumination estimation methods, it does not need to know in advance the scene object geometry and texture information. It has obtained certain research results. Later the researchers used aitificial intelligence method to estimate the scene illumination parameter. But they all need to be trained before use. They can only meet the complex static scene illumination direction estimation. 


\section{References}

[1]. ZHAO Qinping. Overview of virtualreality. Information Sciences.Vol.39 (2009) No. 1, P.2-46.

[2]. LU Yingda.et al. Blind identification for light source images based on inconsistency in light source direction. Journal of Jilin University. Vol.40 (2010) No. 6, P.1673-1677.

[3]. LI Yezhou.et al. Exposing digital image forgeries by detecting inconsistence in light source direction. Journal of Beijing University of Posts and Telecommunications. Vol.34 (2011) No.3, P. 26-30.

[4]. ZHANG Chuang. Research and application of single image light direction estimation algorithm. Video Engineering. Vol. 35(2011) No.15, P.41-45.

[5]. LU Fengshun.et al. Sur-vey of CPU/GPU synergetic parallel computing. Computer Science. Vol.38 (2011) No.35, P.5-9. 